\title{
How Carbon Nanotubes be used in Mechanical Applications
}

\section{Lianxi Zheng*}

School of Mechanical and Aerospace Engineering, Nanyang Technological University 639798, Singapore

Carbon Nano Tubes (CNTs) have excellent electrical and mechanical properties. Their Young's modulus is about $1 \mathrm{TPa}$, and the tensile strength reaches about 11-63 GPa [1-3]. These extraordinary properties make them very attractive for advanced mechanical applications.

At microscopic scale, CNTs have promised bright future for Nano Electro Mechanical Systems (NEMS) through the successful demonstrations on high frequency oscillators [4], rotational actuators [5], nanometer tweezers [6] and nanometer cargoes [7]. These NEMS devices possess unique motion capabilities that are useful for a range of sensing and detection applications. The ultraminiature sensors could provide spatial resolution at the atomic scale and vibrate at frequencies in the gigahertz range, making them possible to measure the forces between individual biomolecules [8], forces arising from magnetic resonance of single spin [9], and perturbations that arise from mass fluctuations involving single atoms and molecules [10]. In addition, high young's modulus and low specific weight of single-walled CNTs qualify them as ultimate mechanical resonators for studying mechanical motion in quantum regime. Strong coupling between single-electron tunneling and nanomechanical motion in CNTs has been observed $[11,12]$.

At macro scale level, great progresses have been made in CNT actuations by coupling CNT's excellent mechanical properties and electrical properties. Large strain and high stress actuators have been studied based upon CNT electrostatic actuation [13], CNT electrochemical actuation [14], and CNT-polymer actuation [15]. Although practical actuators are still under developing, the success of direct conversion of electrical energy into mechanical energy from these actuations offers the opportunities for a number of hightechnology applications, including humanoid robots, artificial and damaged hearts, artificial limbs, medical prosthetic devices, bird- or insect-like air vehicles, etc [16].

Ultimately, it is better to prepare CNTs as structural materials, in order to fully explore their superior mechanical properties. But the dimensions of CNTs, a few nanometers in diameter and up to millimeter in length [17], have confronted us with great challenge in conventional reinforcement approach, i.e., dispersing random CNTs into polymer matrix to fabricate reinforced composite materials. This approach has faced at least two major problems $[18,19]$ : poor dispersion and poor load transfer. Because of van der Waals attractive forces, CNTs, especially for long CNTs that are essential for effective load transfer, tend to aggregate into bundles, making uniform dispersion into a polymer matrix extremely difficult. The non-reactive nature of the CNT's outer wall yields relatively weak bonding at CNT-polymer interface; this in turn gives poor load transfer from matrix to CNTs. As a result, all currently reported experimental data are significantly below theoretical predictions. Even in the future when these two limiting factors are eliminated to some extent, low CNT volume fraction in composites will still limit the performance of the final products. Luckily, recent progresses [20-24] in neat CNT fibers demonstrate the possibility to retain CNT's excellent properties at larger scale and more practicable level. The CNT fibers have been reported to have tensile strength of 1 3 GPa, Young's modulus of 100 260 GPa, toughness of $100 \sim 900 \mathrm{~J} / \mathrm{g}$, and density of $0.2 \mathrm{~g} / \mathrm{cm} 3$. These progresses motivate further study of lightweight and high strength composites for possible structural applications.

Nevertheless, CNT's future still strongly depends on our capabilities on two extremes: controllability of CNT structure at atomic level, and the capability of retaining the properties of individual CNTs at assembly level. Particularly for CNT assemblies, which are still much poor in performance compared with individual CNTs, future research need to focus on the understanding of the failure mechanism of CNT assemblies, aiming at finding key limiting factors and thus providing reliable and high performance CNT assemblies for practical applications.

\section{References}

1. Yu MF, Lourie O, Dyer MJ, Moloni K, Kelly TF, et al. (2000) Strength and breaking mechanism of multiwalled carbon nanotubes under tensile load. Science 287: 637-640.

2. Yu MF, Files BS, Arepalli S, Ruoff RS (2000) Tensile loading of ropes of single wall carbon nanotubes and their mechanical properties. Phys Rev Lett 84 5552-5555.

3. Demczyk BG, Wang YM, Cuming J, Hetman M, Han W, et al. (2002) Direct mechanical measurement of the tensile strength and elastic modulus of multiwalled carbon nanotubes. Mater Sci Eng 334: 173-178.

4. Sazonova V, Yaish Y, Ustünel H, Roundy D, Arias TA, et al. (2004) A tunable carbon nanotube electromechanical oscillator. Nature 431: 284-287.

5. Fennimore AM, Yuzvinsky TD, Han WQ, Fuhrer MS, Cumings J, et al. (2003) Rotational actuators based on carbon nanotubes. Nature 424: 408-410.

6. Kim P, Lieber CM (1999) Nanotube nanotweezers. Science 286: 2148-2150.

7. Barreiro A, Rurali R, Hernández ER, Moser J, Pichler T, et al. (2008) Subnanometer motion of cargoes driven by thermal gradients along carbon nanotubes. Science 320: 775-778.

8. Bustamante C, Chemla YR, Forde NR, Izhaky D (2004) Mechanical processes in biochemistry. Annu Rev Biochem 73: 705-748.

9. Rugar D, Budakian R, Mamin HJ, Chui BW (2004) Single spin detection by magnetic resonance force microscopy. Nature 430: 329-332.

10. Yang YT, Callegari C, Feng XL, Ekinci KL, Roukes ML (2006) Zeptogram-scale nanomechanical mass sensing. Nano Lett 6: 583-586.

11. Steele GA, Hüttel AK, Witkamp B, Poot M, Meerwaldt HB, et al. (2009) Strong coupling between single-electron tunneling and nanomechanical motion. Science 325: 1103-1107.

12. Lassagne B, Tarakanov Y, Kinaret J, Garcia-Sanchez D, Bachtold A (2009) Coupling mechanics to charge transport in carbon nanotube mechanical resonators. Science 325: 1107-1110.

13. Aliev AE, Oh J, Kozlov ME, Kuznetsov AA, Fang S, et al. (2009) Giant-stroke, superelastic carbon nanotube aerogel muscles. Science 323: 1575-1578.

*Corresponding author: Lianxi Zheng, School of Mechanical and Aerospace Engineering, Nanyang Technological University 639798, Singapore, E-mail: Lxzheng@ntu.edu.sg

Received August 21, 2012; Accepted August 23, 2012; Published August 25 2012

Citation: Zheng L (2012) How Carbon Nanotubes be used in Mechanical Applications. J Appl Mech Eng 1:e108. doi:10.4172/2168-9873.1000e108

Copyright: (c) 2012 Zheng L. This is an open-access article distributed under the terms of the Creative Commons Attribution License, which permits unrestricted use, distribution, and reproduction in any medium, provided the original author and source are credited. 
14. Baughman RH, Cui C, Zakhidov AA, Iqbal Z, Barisci JN, et al. (1999) Carbon nanotube actuators. Science 284: 1340-1344.

15. Koerner H, Price G, Pearce NA, Alexander M, Vaia RA (2004) Remotely actuated polymer nanocomposites--stress-recovery of carbon-nanotube-filled thermoplastic elastomers. Nat Mater 3: 115-120.

16. Baughman RH (2005) Materials science. Playing nature's game with artificial muscles. Science 308: 63-65.

17. Zheng LX, O'Connell MJ, Doorn SK, Liao XZ, Zhao YH, et al. (2004) Ultralong single-wall carbon nanotubes. Nat Mater 3: 673-676.

18. Szleifer I, Yerushalmi-Rozen R (2005) Polymers and carbon nanotubesdimensionality, interactions and nanotechnology. Polymer 46: 7803-7818.

19. Fiedler B, Gojny FH, Wichmann MHG, Wichmann CMN, Schulte K (2006)
Fundamental aspects of nano-reinforced composites. Compos Sci Technol 66: 3115-3125.

20. Zhang X, Li Q, Holesinger TG, Arendt PN, Huang J, et al. (2007) Ultrastrong, stiff, and lightweight carbon-nanotube fibers. Adv Mater 19: 4198-4201.

21. Zhang X, Li Q, Tu Y, Li Y, Coulter JY, et al. (2007) Strong carbon-nanotube fibers spun from long carbon-nanotube arrays. Small 3: 244-248.

22. Li QW, Zhang XF, DePaula RF, Zheng LX, Zhao YH, et al. (2006) Sustained growth of long carbon nanotube arrays for fiber spinning. Adv Mater 18: 31603163.

23. Zheng L, Sun G, Zhan Z (2010) Tuning array morphology for high-strength carbon-nanotube fibers. Small 6: 132-137.

24. Zhang YN, Zheng LX, SunGZ, Zhan ZY, Liao K (2012) Failure mechanisms of carbon nanotube fibers under different strain rates. Carbon 50: 2887-2893. 